\title{
IB-DFE Receivers with Space Diversity for CP-Assisted DS-CDMA and MC-CDMA Systems
}

\author{
Rui Dinis ${ }^{1 *}$, Paulo Silva ${ }^{2}$ and António Gusmão ${ }^{3}$ \\ ${ }^{1}$ ISR-IST, Tech. Univ. of Lisbon, Portugal \\ ${ }^{2}$ ISR-IST/EST, Univ. of Algarve, Portugal \\ ${ }^{3}$ CAPS-IST, Tech. Univ. of Lisbon, Portugal
}

\begin{abstract}
SUMMARY
MC-CDMA (MultiCarrier Code Division Multiple Access), currently regarded as a promising multiple access scheme for broadband communications, is known to combine the advantages of an OFDM-based (Orthogonal Frequency Division Multiplexing), CP-assisted (Cyclic Prefix) block transmission with those of CDMA systems. Recently, it was recognized that DS-CDMA (Direct Sequence) implementations can also take advantage of the benefits of the CP-assisted block transmission approach, therefore enabling an efficient use of FFT-based (Fast Fourier Transform), chip level FDE (Frequency-Domain Equalization) techniques.

When employing a linear FDE with both MC-CDMA and DS-CDMA, the FDE coefficients can be optimized under the MMSE criterion (Minimum Mean Squared Error), so as to avoid significant noise enhancement. The residual interference levels can be very high, especially for fully loaded scenarios, since the FDE/MMSE does not perform a perfect channel inversion.

This paper deals with CP-assisted DS-CDMA systems and MC-CDMA systems with frequencydomain spreading. We consider the use of IB-DFE (Iterative Block Decision Feedback Equalization) FDE techniques as an alternative to conventional, linear FDE techniques, and derive the appropriate IB-DFE parameters in a receiver diversity context. Our performance results show that IB-DFE techniques with moderate complexity allow significant performance gains in both systems, with BER (Bit Error Rate) that can be close to the single-code matched filter bound (especially for the CP-assisted DS-CDMA alternative), even with full code usage.
\end{abstract}

Keywords: CDMA (Code Division Multiple Access), Cyclic Prefix, Frequency-Domain Equalization, Iterative Decision-Feedback Approach, Receiver Diversity.

Copyright (c) 2007 AEIT

\section{Introduction}

It is widely known that a CP-assisted block transmission approach, allowing low-complexity FDE receiver techniques, is suitable for high data rate transmission over severely time-dispersive channels. This approach can be employed with either MC (MultiCarrier) or SC (Single-Carrier) modulations

*Correspondence to: IST, Tech. Univ. of Lisbon, Av. Rovisco Pais, 1049-001 Lisboa, Portugal. E-mail: rdinis@ist.utl.pt

Copyright (c) 2007 AEIT

Prepared using ettauth.cls [Version: 2007/01/05 v1.00]
$[1,2]$. When adopted in CDMA systems, it leads to MC-CDMA implementations [3, 4, 5], and also, as recently recognized, quite efficient DS-CDMA implementations [6, 7]. These CP-assisted schemes are especially interesting for multicode and/or downlink transmission, taking advantage of synchronized, orthogonal spreading codes. In fact, since all spreading codes face the same channel, the multicode detection can be efficiently treated as an equalization problem. Although these leads to suboptimum receiver designs, their complexity is much lower than the optimum 
receivers (whose complexity grows exponentially with the number of spreading codes [8, 9, 10]).

Conventional, linear FDE techniques are known to lead to a significant noise enhancement when a ZF (Zero Forcing) criterion is adopted for restoring orthogonality in channels with deep in-band notches. A simple frequency-domain matched filtering is also known to lead to a very poor performance. For this reason, an MMSE (Minimum Mean-Squared Error) FDE equalizer is usually preferable [11]. However, an FDE/MMSE does not perform an ideal channel inversion; therefore, when this type of equalizer is employed within CP-assisted CDMA systems, we are not able to keep the different spreading codes fully orthogonalized. This means severe interference levels, especially when different powers are assigned to different codes.

It is well-known that nonlinear equalizers can significantly outperform linear equalizers. For this reason, a promising IB-DFE (Iterative Block Decision Feedback Equalization) approach was proposed for CP-assisted SC schemes [12], with both the feedforward and the feedback parts implemented in the frequency domain (a similar concept was also proposed in [13]). An extension of this approach to $\mathrm{SC} / \mathrm{FDE}$ receivers with space diversity was also shown to be feasible [14], allowing much better performance than the conventional, linear SC/FDE receiver approach. An appropriate extension to layered space-time SC/FDE receivers for multiple antenna systems was also developed [15, 16].

This paper deals with CP-assisted MC-CDMA systems, with frequency-domain spreading, and DSCDMA systems, by considering the use of IBDFE techniques in space diversity receivers, as an alternative to conventional linear FDE techniques. For MC-CDMA schemes, our receiver design is related to the turbo receiver proposed in [17], although with a much lower signal processing complexity since we do not employ the channel decoder output in the feedback loop. Our receiver for DS-CDMA is related to the turbo receiver proposed in [18], however, with much lower signal processing complexity, especially when severely time-dispersive channels are considered due to the fact that we are considering an FFTbased frequency-domain implementation (moreover, the channel decoder output is required for the feedback loop in [18]). Moreover, we consider the receiver design with $L$-order space diversity, while that was not considered in [17] and [18].
The paper is organized as follows: Sec. 2 describes the CP-assisted CDMA schemes to be considered and the basic linear FDE principles. The IB-DFE receiver techniques are addressed in sec. 3, where their parameters are derived. A set of performance results, in the CDMA context, is presented in sec. 4, and sec. 5 is concerned with the conclusions and complementary remarks of this paper.

\section{CP-Assisted CDMA Systems with Linear FDE}

In this section we describe the CP-assisted DS-CDMA and MC-CDMA systems to be considered, involving a multicode transmission with constant spreading factor (the extension to VSF schemes (Variable Spreading Factor) [19]) is straightforward). In both cases, the receiver can be based on a linear FDE (see fig. $1 \mathrm{~A}$, where an $L$-branch space diversity receiver is considered). As with other CP-assisted techniques, after removing the cyclic extension, the received timedomain block associated to each diversity branch, $\left\{y_{n}^{(l)} ; n=0,1, \ldots, N-1\right\}, l=1,2, \ldots, L$, is passed to the frequency domain, leading to the block $\left\{Y_{k}^{(l)} ; k=\right.$ $0,1, \ldots, N-1\}$, with $N$ denoting the length of the useful part of the block ${ }^{\dagger}$. When the cyclic extension is longer than the overall channel impulse response, the samples $Y_{k}^{(l)}$ can be written as

$$
Y_{k}^{(l)}=H_{k}^{(l)} S_{k}+N_{k}^{(l)},
$$

where $H_{k}^{(l)}$ and $N_{k}^{(l)}$ denote the channel frequency response and the noise term for the $k$ th frequency and the $l$ th diversity branch, respectively, and $\left\{S_{k} ; k=\right.$ $0,1, \ldots, N-1\}=\operatorname{DFT}\left\{s_{n} ; n=0,1, \ldots, N-1\right\}$, with $\left\{s_{n} ; n=0,1, \ldots, N-1\right\}$ denoting the transmitted time-domain block. For a linear FDE, the frequency-domain samples at its output are given by

$$
\tilde{S}_{k}=\sum_{l=1}^{L} F_{k}^{(l)} Y_{k}^{(l)}
$$

where the set $\left\{F_{k}^{(l)} ; k=0,1, \ldots, N-1\right\}$ denotes the FDE coefficients associated to the $l$ th diversity branch. By setting

$$
F_{k}^{(l)}=\frac{H_{k}^{*(l)}}{\sum_{l=1}^{L}\left|H_{k}^{(l)}\right|^{2}},
$$

${ }^{\dagger}$ For MC-CDMA $N$ is the number of subcarriers; for DS-CDMA $N$ is the number of chips per block. 



Figure 1. Linear FDE receiver structure with $L$-branch space diversity (A) and transmission models for MC-CDMA (B) and DS-CDMA (C).

we could invert completely the channel effects (ZF criterion) while actually implementing an approximate MRC (Maximal Ratio Combining) rule. As an alternative, we could optimize these coefficients under the MMSE criterion (Minimum Mean Squared Error), leading to $[11]$

$$
F_{k}^{(l)}=\frac{H_{k}^{*(l)}}{\alpha+\sum_{l=1}^{L}\left|H_{k}^{(l)}\right|^{2}},
$$

where

$$
\alpha=\frac{\sigma_{N}^{2}}{\sigma_{S}^{2}}
$$

with $\sigma_{N}^{2}$ denoting the variance of the noise terms (supposed to be identical in all diversity branches) and $\sigma_{S}^{2}$ denoting the variance of the data symbols.

\subsection{MC-CDMA}

Let us consider an MC-CDMA scheme. The frequency-domain block to be transmitted is $\left\{S_{k} ; k=\right.$ $0,1, \ldots, N-1\}$, where $N=K M$, with $K$ denoting the spreading factor and $M$ the number of data symbols per spreading code. The frequency-domain symbols are given by

$$
S_{k}=\sum_{p=1}^{P} \xi_{p} S_{k, p},
$$

where $P$ is the number of spreading codes and $\xi_{p}$ is an appropriate weighting coefficient for power control purposes (the power associated to the $p$ th spreading code is proportional to $\left.\xi_{p}^{2}\right) .\left\{S_{k, p} ; k=0,1, \ldots, N-1\right\}$ is an interleaved version of $\left\{S_{k, p}^{\prime} ; k=0,1, \ldots, N-1\right\}$ (rectangular $K \times M$ interleaver, so that different chips associated with a given data symbol are spaced by $M$ subcarriers).

$$
S_{k, p}^{\prime}=C_{k, p} A_{\lfloor k / K\rfloor, p}
$$

is the $k$ th chip for the $p$ th spreading code $\left(\lfloor x\rfloor\right.$ denotes 'larger integer not higher that $\left.x^{\prime}\right)$. $\left\{A_{m, p} ; m=0,1, \ldots, M-1\right\}$ denotes the block of data symbols associated to the $p$ th spreading code and $\left\{C_{k, p} ; k=0,1, \ldots, N-1\right\}$ is the corresponding spreading sequence. An orthogonal spreading is assumed throughout this paper, with $C_{k, p}$ belonging to a QPSK constellation (Quaternary Phase Shift 
Keying). Without loss of generality, it is assumed that $\left|C_{k, p}\right|=1$. At the receiver side, the $A_{k, p}$ coefficients are estimated from

$$
\tilde{A}_{m, p}=\sum_{k^{\prime} \in \Psi_{m}} \tilde{S}_{k^{\prime}} C_{k^{\prime}, p}^{*},
$$

with $\Psi_{m}=\{m, m+M, \ldots, m+(K-1) M\}$ denoting the set of frequencies employed to transmit the $m$ th data symbol of each spreading code and $\tilde{S}_{k}$ given by (2) (see fig. 1B). The data estimates $\hat{A}_{m, p}$ are the "hard decisions" associated to $\tilde{A}_{m, p}$.

\subsection{DS-CDMA}

Let us consider now a DS-CDMA scheme. The transmitted block of chips is $\left\{s_{n} ; n=0,1, \ldots, N-1\right\}$, once again $N=K M, K$ is the spreading factor and $M$ is the number of data symbols for each spreading code. The overall "chip" symbols $s_{n}$ are given by

$$
s_{n}=\sum_{p=1}^{P} \xi_{p} s_{n, p},
$$

where $\xi_{p}$ is an weighting coefficient, proportional to the transmitted power for the $p$ th user, and

$$
s_{n, p}=c_{n, p} a_{\lfloor n / K\rfloor, p}
$$

is the $n$th chip for the $p$ th user. $\left\{a_{m} ; m=\right.$ $0,1, \ldots, M-1\}$ denotes the block of data symbols associated to the $p$ th user and $\left\{c_{n, p} ; n=0,1, \ldots, N-\right.$ $1\}$ denotes the corresponding spreading sequence. As with MC-CDMA, an orthogonal spreading and $\left|c_{n, p}\right|=1$ are assumed.

In this case, the FDE receiver could estimate the data symbols from

$$
\tilde{a}_{m, p}=\sum_{n^{\prime}=m K}^{m+K-1} \tilde{s}_{n^{\prime}} c_{n^{\prime}, p}^{*},
$$

with $\left\{\tilde{s}_{n} ; n=0,1, \ldots, N-1\right\}=\operatorname{IDFT}\left\{\tilde{S}_{k} ; k=\right.$ $0,1, \ldots, N-1\}$ (see fig. $1 \mathrm{C}$ ). The data estimates $\hat{a}_{m, p}$ are the "hard decisions" associated to $\tilde{a}_{m, p}$.

\section{Iterative Block Decision Feedback Equalization for} CP-Assisted CDMA

\subsection{Receiver Structure}

Fig. 2 presents the receiver structures that we are considering in this paper, where the linear FDE is replaced by an IB-DFE. In both cases, for a given iteration $i$, the output samples are given by

$$
\tilde{S}_{k}^{(i)}=\sum_{l=1}^{L} F_{k}^{(l, i)} Y_{k}^{(l)}-B_{k}^{(i)} \hat{S}_{k}^{(i-1)}
$$

where $\quad\left\{F_{k}^{(l, i)} ; k=0,1, \ldots, N-1\right\} \quad(l=1,2, \ldots, L)$ and $\left\{B_{k}^{(i)} ; k=0,1, \ldots, N-1\right\}$ denote the feedforward and the feedback equalizer coefficients, respectively, optimized so as to maximize the "overall signal-tonoise plus interference", as described in the following. The block $\left\{\hat{S}_{k}^{(i-1)} ; k=0,1, \ldots, N-1\right\}$ is an estimate of the transmitted block $\left\{S_{k}^{(i-1)} ; k=0,1, \ldots, N-1\right\}$, obtained form the data estimates of the $(i-1)$ th iteration, $\left\{\hat{A}_{k, p}^{(i-1)} ; k=0,1, \ldots, M-1\right\}$ in the MCCDMA case and IDFT $\left\{\hat{a}_{n, p}^{(i-1)} ; n=0,1, \ldots, M-1\right\}$ in the DS-CDMA case, as in (6)-(7) or (9)-(10), respectively.

The data estimates are the hard-decisions associated to the despreaded samples, $\left\{\tilde{A}_{k, p}^{(i-1)} ; k=\right.$ $0,1, \ldots, M-1\}$ in the MC-CDMA case and $\left\{\tilde{a}_{n, p}^{(i-1)} ; n=0,1, \ldots, M-1\right\} \quad$ in the DS-CDMA case, are given by (8) and (11), respectively. It should be pointed out that "soft estimates" could be employed in the feedback loop instead of "hard estimates"; since the performances are similar, unless we use the channel decoder output in the feedback loop [20], we just considered "hard estimates".

\subsection{Computation of the Receiver Parameters}

If there were no intersymbol interference (ISI) at the output of the feedforward filter, the "overall" channel frequency response $\sum_{l=1}^{L} F_{k}^{(l, i)} H_{k}^{(l)}$ would be constant. Therefore, the ISI component in the frequency domain is associated to the difference between the average channel frequency response after the feedforward filter, defined as

$$
\gamma^{(i)}=\frac{1}{N} \sum_{k=0}^{N-1} \sum_{l=1}^{L} F_{k}^{(l, i)} H_{k}^{(l)},
$$

and its actual value. If we have reliable estimates of the transmitted block, the feedback filter can then be used to remove this residual ISI.

Therefore, the equalized frequency-domain samples associated to each iteration, $\tilde{S}_{k}^{(i)}$, can be written as

$$
\tilde{S}_{k}^{(i)}=\gamma^{(i)} S_{k}+\varepsilon_{k}^{E q(i)}
$$




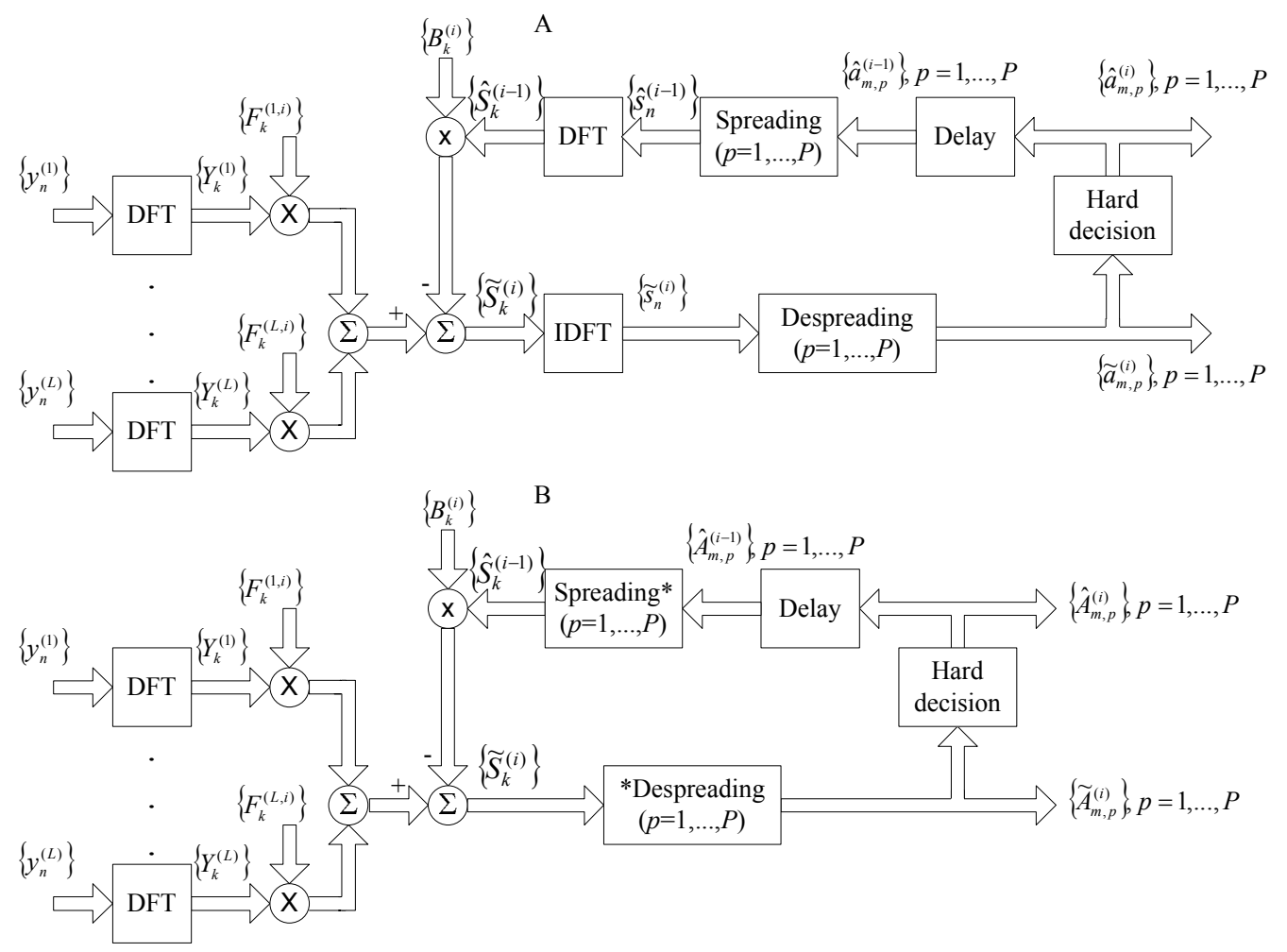

Figure 2. IB-DFE receiver for MC-CDMA (A) (* denotes the complementary interleaving/deinterleaving) and DSCDMA (B).

where $\varepsilon_{k}^{E q(i)}=\tilde{S}_{k}^{(i)}-\gamma^{(i)} S_{k}$ denotes an overall error that includes both the channel noise and the residual ISI. In the same way, the corresponding time-domain samples can be written as

$$
\tilde{s}_{n}^{(i)}=\gamma^{(i)} s_{n}+\varepsilon_{n}^{e q(i)}
$$

where the block $\left\{\varepsilon_{n}^{e q(i)} ; n=0,1, \ldots, N-1\right\}$ is the IDFT of the block $\left\{\varepsilon_{k}^{E q(i)} ; k=0,1, \ldots, N-1\right\}$.

The forward and backward IB-DFE coefficients, $\left\{F_{k}^{(l, i)} ; k=0,1, \ldots, N-1\right\} \quad(l=1,2, \ldots, L) \quad$ and $\left\{B_{k}^{(i)} ; k=0,1, \ldots, N-1\right\}$, respectively, are chosen so as to maximize the "signal-to-noise plus interference ratio", SNIR, defined as

$$
S N I R^{(i)}=\frac{\left|\gamma^{(i)}\right|^{2} E\left[\left|S_{k}\right|^{2}\right]}{E\left[\left|\varepsilon_{k}^{E q(i)}\right|^{2}\right]} .
$$

The frequency-domain estimates, $\hat{S}_{k}^{(i)}$, can be written as

$$
\hat{S}_{k}^{(i)}=\rho^{(i)} S_{k}+\Delta_{k}^{(i)},
$$

where the correlation coefficient $\rho^{(i)}$ is given by

$$
\rho^{(i)}=\frac{E\left[\hat{s}_{n}^{(i)} s_{n}^{*}\right]}{E\left[\left|s_{n}\right|^{2}\right]}=\frac{E\left[\hat{S}_{k}^{(i)} S_{k}^{*}\right]}{E\left[\left|S_{k}\right|^{2}\right]}
$$

and $\Delta_{k}^{(i)}$ denotes a zero-mean error term. Since it is assumed that $E\left[\Delta_{k}^{(i)} S_{k^{\prime}}^{(i) *}\right] \approx 0$ for $k^{\prime} \neq k$,

$$
E\left[\left|\Delta_{k}^{(i)}\right|^{2}\right] \approx\left(1-\left(\rho^{(i)}\right)^{2}\right) E\left[\left|S_{k}\right|^{2}\right]
$$

The coefficient $\rho^{(i-1)}$, which can be regarded as the blockwise reliability of the decisions used in the feedback loop (from the previous iteration), is crucial for the good performance of the proposed receivers, can be estimated from the samples $\tilde{a}_{n, p}$ as described in the next subsection. 
By combining (1), (12) and (17), we obtain

$$
\begin{aligned}
\tilde{S}_{k}^{(i)}= & \sum_{l=1}^{L} F_{k}^{(l, i)}\left(H_{k}^{(l)} S_{k}+N_{k}^{(l)}\right) \\
& \quad-B_{k}^{(i)}\left(\rho^{(i-1)} S_{k}+\Delta_{k}^{(i-1)}\right) \\
= & \gamma^{(i)} S_{k} \\
& +\left(\sum_{l=1}^{L} F_{k}^{(l, i)} H_{k}^{(l)}-\gamma^{(i)}-\rho^{(i-1)} B_{k}^{(i)}\right) S_{k} \\
& -B_{k}^{(i)} \Delta_{k}^{(i-1)}+\sum_{l=1}^{L} F_{k}^{(l, i)} N_{k}^{(l)}
\end{aligned}
$$

This means that $\tilde{S}_{k}^{(i)}$ has four terms: a "signal" component, $\gamma^{(i)} S_{k}$, and three "noise" components. The first component in the last equality of (20) is the residual intersymbol interference; the second component accounts for the errors in $\hat{s}_{n}^{(i-1)}$; and the final component is concerned to the channel noise.

The maximization of the SNIR (16) is equivalent to the minimization of

$$
\begin{aligned}
& E\left[\left|\varepsilon_{k}^{E q(i)}\right|^{2}\right]= \\
& =E\left[\left|\sum_{l=1}^{L} F_{k}^{(l, i)} H_{k}^{(l)}-\gamma^{(i)}-\rho^{(i-1)} B_{k}^{(i)}\right|^{2}\right] E\left[\left|S_{k}\right|^{2}\right] \\
& +E\left[\left|B_{k}^{(i)} \Delta_{k}^{(i-1)}\right|^{2}\right]+E\left[\left|\sum_{l=1}^{L} F_{k}^{(l, i)} N_{k}^{(l)}\right|^{2}\right] \\
& =E\left[\left|\sum_{l=1}^{L} F_{k}^{(l, i)} H_{k}^{(l)}-\gamma^{(i)}-\rho^{(i-1)} B_{k}^{(i)}\right|^{2}\right] 2 \sigma_{S}^{2} \\
& +E\left[\left|B_{k}^{(i)}\right|^{2}\right]\left(1-\left(\rho^{(i-1)}\right)^{2}\right) 2 \sigma_{S}^{2} \\
& +\sum_{l=1}^{L} E\left[\left|F_{k}^{(l, i)}\right|^{2}\right] 2 \sigma_{N}^{2},
\end{aligned}
$$

conditioned to a given $\gamma^{(i)}$, where $2 \sigma_{S}^{2}=E\left[\left|S_{k}\right|^{2}\right]$. The optimum receiver coefficients can be obtained by employing the Lagrangian's multipliers method. For this purpose, we can define the Lagrangian function

$J=E\left[\left|\varepsilon_{k}^{E q(i)}\right|^{2}\right]+\lambda^{(i)}\left(\frac{1}{N} \sum_{k=0}^{N-1} \sum_{l=1}^{L} F_{k}^{(l, i)} H_{k}^{(l)}-1\right)$,

and assume that the optimization is carried out under $\gamma^{(i)}=1$. The optimum receiver coefficients are obtained by solving the following set of $L+2$ equations

$$
\begin{aligned}
& \frac{\partial J}{\partial F_{k}^{(l, i)}}= \\
& =4 \sigma_{S}^{2} H_{k}^{(l) *}\left(\sum_{l^{\prime}=1}^{L} F_{k}^{\left(l^{\prime}, i\right)} H_{k}^{\left(l^{\prime}\right)}-1-\rho^{(i-1)} B_{k}^{(i)}\right. \\
& \left.\quad+\frac{\lambda^{(i)}}{2 \sigma_{S}^{2} N}\right)+4 \sigma_{N}^{2} F_{k}^{(l, i)}=0, \quad l=1,2, \ldots, L \\
& \frac{\partial J}{\partial B_{k}^{(i)}}= \\
& =-4 \sigma_{S}^{2} \rho^{(i-1)}\left(\sum_{l^{\prime}=1}^{L} F_{k}^{\left(l^{\prime}, i\right)} H_{k}^{\left(l^{\prime}\right)}-1-\rho^{(i-1)} B_{k}^{(i)}\right) \\
& \quad+4 \sigma_{S}^{2}\left(1-\left(\rho^{(i-1)}\right)^{2}\right) B_{k}^{(i)}=0
\end{aligned}
$$

and

$$
\frac{\partial J}{\partial \lambda^{(i)}}=\frac{1}{N} \sum_{k=0}^{N-1} \sum_{l=1}^{L} F_{k}^{(l, i)} H_{k}^{(l)}-1=0 .
$$

As expected, (25) is equivalent to $\gamma^{(i)}=1$. The remaining equations can be rewritten in the form

$$
\begin{aligned}
& H_{k}^{(l) *}\left(\sum_{l^{\prime}=1}^{L} F_{k}^{\left(l^{\prime}, i\right)} H_{k}^{\left(l^{\prime}\right)}-1-\rho^{(i-1)} B_{k}^{(i)}\right. \\
& \left.\quad+\frac{\lambda^{(i)}}{2 \sigma_{S}^{2} N}\right)+\alpha F_{k}^{\left(l^{\prime}, i\right)}=0, \quad l=1,2, \ldots, L
\end{aligned}
$$

and

$$
\begin{aligned}
& \rho^{(i-1)}\left(\sum_{l^{\prime}=1}^{L} F_{k}^{\left(l^{\prime}, i\right)} H_{k}^{\left(l^{\prime}\right)}-1-\rho^{(i-1)} B_{k}^{(i)}\right)= \\
& =\left(1-\left(\rho^{(i-1)}\right)^{2}\right) B_{k}^{(i)},
\end{aligned}
$$

with $\alpha$ given by (5).

From (27), the optimum values of $B_{k}^{(i)}$ are

$$
B_{k}^{(i)}=\rho^{(i-1)}\left(\sum_{l^{\prime}=1}^{L} F_{k}^{\left(l^{\prime}, i\right)} H_{k}^{\left(l^{\prime}\right)}-1\right) .
$$

By replacing (28) in (26), we get the set of $L$ equations

$$
\begin{aligned}
(1 & \left.-\left(\rho^{(i-1)}\right)^{2}\right) H_{k}^{(l) *} \sum_{l^{\prime}=1}^{L} F_{k}^{\left(l^{\prime}, i\right)} H_{k}^{\left(l^{\prime}\right)}+\alpha F_{k}^{(l, i)} \\
& =\left(1-\left(\rho^{(i-1)}\right)^{2}-\frac{\lambda^{(i)}}{2 \sigma_{S}^{2} N}\right) H_{k}^{(l) *}, \quad l=1,2, \ldots, L .
\end{aligned}
$$


It can be easily verified by substitution that the solutions of (26) are

$$
\begin{aligned}
F_{k}^{(l, i)} & =\frac{K_{F}^{(i)} H_{k}^{(l) *}}{\alpha+\left(1-\left(\rho^{(i-1)}\right)^{2}\right) \sum_{l^{\prime}=1}^{L}\left|H_{k}^{\left(l^{\prime}\right)}\right|^{2}}, \\
l=1,2, \ldots, L, &
\end{aligned}
$$

where the normalization constant

$$
K_{F}^{(i)}=1-\left(\rho^{(i-1)}\right)^{2}-\frac{\lambda^{(i)}}{2 \sigma_{S}^{2} N}
$$

ensures that $\gamma^{(i)}=1$.

These feedforward coefficients can be used in (28) for obtaining the feedback coefficients $B_{k}^{(i)}$.

Clearly, for the first iteration $(i=0)$, no information exists about $S_{k}$ and the correlation coefficient in (30) is zero. This means that $B_{k}^{(0)}=0$ and

$$
F_{k}^{(l, 0)}=\frac{K_{F}^{(0)} H_{k}^{(l) *}}{\alpha+\sum_{l^{\prime}=1}^{L}\left|H_{k}^{\left(l^{\prime}\right)}\right|^{2}}, \quad l=1,2, \ldots, L,
$$

corresponding to the optimum frequency-domain equalizer coefficients under the MMSE criterion (Minimum Mean-Squared Error) [11, 21]. After that first iteration, and if the residual BER is not too high (at least for the spreading codes with higher transmit power), we can use the feedback coefficients to eliminate a significant part of the residual interference. When $\rho \approx 1$ (after several iterations and/or moderate-to-high SNRs), we have an almost full cancellation of the "inter-code" interference through these coefficients, while the feedforward coefficients perform an approximate matched filtering.

It should be noted that, when $L=1$ (no diversity) the IB-DFE parameters derived above become identical to those given in [12]. It should also be noted that the feedforward coefficients can take the form

$$
F_{k}^{(l, i)}=H_{k}^{(l) *} G_{k}^{(i)}, l=1,2, \ldots, L,
$$

with

$$
G_{k}^{(i)}=\frac{K_{F}^{(i)}}{\alpha+\left(1-\left(\rho^{(i-1)}\right)^{2}\right) \sum_{l^{\prime}=1}^{L}\left|H_{k}^{\left(l^{\prime}\right)}\right|^{2}} .
$$

This means that the bank of feedforward filters can be replaced by a bank of matched filters which implement an ideal MRC, followed by a single feedforward filter characterized by the set of coefficients $\left\{G_{k}^{(i)} ; k=\right.$ $0,1, \ldots, N-1\}$.

\subsection{Calculation of $\rho_{p}$}

In this subsection we show how one can obtain an estimate of the correlation coefficient. Assuming uncorrelated data blocks, it can be easily shown that

$$
\rho^{(i-1)}=\sum_{p=1}^{P} \xi_{p}^{2} \rho_{p}^{(i-1)},
$$

with

$$
\rho_{p}^{(i-1)}=\frac{E\left[\hat{A}_{k, p} A_{k, p}^{*}\right]}{E\left[\left|A_{k, p}\right|^{2}\right]}=\frac{E\left[\hat{a}_{n, p} a_{n, p}^{*}\right]}{E\left[\left|a_{n, p}\right|^{2}\right]},
$$

denoting the correlation coefficient associated to the $p$ th user. For a DS-CDMA scheme $\rho_{p}^{(i)}$ can be obtained as follows (a similar approach could be employed for MC-CDMA schemes).

Let us assume that the transmitted symbols $a_{n, p}$ belong to a QPSK constellation (the generalization to other constellations is straightforward). In this case,

$$
a_{n, p}=a_{n, p}^{I}+j a_{n, p}^{Q}= \pm d \pm j d
$$

where $a_{n, p}^{I}=\operatorname{Re}\left\{a_{n, p}\right\} \quad$ and $\quad a_{n, p}^{Q}=\operatorname{Im}\left\{a_{n, p}\right\} \quad$ are the in-phase and quadrature components of $a_{n, p}$, respectively, and $d=D / 2$, with $D$ corresponding to the minimum Euclidean distance (for the sake of simplicity, in the following we will ignore the dependency with the iteration number $i$ ). In this case,

$$
E\left[\left|a_{n, p}\right|^{2}\right]=\frac{D^{2}}{4} .
$$

For an unbiased $\operatorname{FDE}(\gamma=1)$, the time-domain samples at the output of the FDE are

$$
\tilde{a}_{n, p}=\tilde{a}_{n, p}^{I}+j \tilde{a}_{n, p}^{Q}=a_{n, p}+\nu_{n, p},
$$

where $\tilde{a}_{n, p}^{I}=\operatorname{Re}\left\{\tilde{a}_{n, p}\right\}, \tilde{a}_{n, p}^{Q}=\operatorname{Im}\left\{\tilde{a}_{n, p}\right\}$ and $\nu_{n, p}$ is the overall noise component. We will assume that $\nu_{n, p}$ is approximately Gaussian-distributed ${ }^{\ddagger}$, with $E\left[\nu_{n, p}\right]=0$. Moreover, the SNR for detection purposes is

$$
S N I R_{p}^{e q}=\frac{E\left[\left|a_{n, p}\right|^{2}\right]}{E\left[\left|\nu_{n, p}\right|^{2}\right]}=\frac{P\left|\xi_{p}\right|^{2}}{\sum_{p^{\prime}=1}^{P}\left|\xi_{p^{\prime}}\right|^{2}} S N I R,
$$

¥This assumption is reasonable under severely time-dispersive channel conditions. 
with SNIR given by (16), i.e., $S N I R_{p}$ is higher for the users with higher assigned power.

The symbol estimates can be written as

$$
\hat{a}_{n}=a_{n, p}+\varepsilon_{n, p}^{I}+j \varepsilon_{n, p}^{Q}
$$

where the error coefficients $\varepsilon_{n, p}^{I}\left(\right.$ or $\left.\varepsilon_{n, p}^{Q}\right)$ are zero if there is no error in $a_{n, p}^{I}$ (or $a_{n, p}^{Q}$ ) and $\pm D$ otherwise. This means that $\varepsilon_{n, p}^{I}$ and $\varepsilon_{n, p}^{Q}$ are random variables, both taking the values 0 and $\pm D$ with probabilities $1-P_{e, p}$ and $P_{e, p}$, respectively. Therefore,

$$
\rho_{p}=1-2 P_{e, p}
$$

where $P_{e, p}$ denotes the BER associated to the $p$ th user, which can be approximated by

$$
P_{e, p} \approx Q\left(\sqrt{S N I R_{p}^{e q}}\right)
$$

for QPSK constellations.

\section{Performance Results}

In this section we present a set of performance results concerning the proposed receiver structure. We consider the downlink transmission, with each spreading code intended to a given user. It is assumed that $N=256$ (similar results could be obtained for other high values of $N$ ) and the data symbols are selected from a QPSK constellation under a Gray mapping rule. For both DS-CDMA and MC-CDMA, we consider an orthogonal spreading (Hadamard-Walsh sequences plus pseudo-random scrambling sequences with the same chip rate) and the power amplifier at the transmitter is assumed to be linear. The radio channel is characterized by the power delay profile type $\mathrm{C}$ for HIPERLAN/2 (HIgh PERformance Local Area Network) [22], with uncorrelated Rayleigh fading on the different paths. The subcarrier separation is $0.2 \mathrm{MHz}$. Perfect synchronization and channel estimation are assumed in all cases. The number of users is $P=K$, i.e., we are assuming a fully loaded system. For the sake of comparisons, we included the MFB performance (Matched Filter Bound), defined as

$$
P_{b, M F B}=E\left[Q\left(\sqrt{\frac{2 E_{b}}{N_{0}} \frac{1}{N} \sum_{k} \sum_{l}\left|H_{k}^{(l)}\right|^{2}}\right)\right],
$$

where the expectation is taken over a large number of channels and $E\left[\left|H_{k}^{(l)}\right|^{2}\right]=1$. For MC-CDMA systems, the optimum SU performance (Single User), achievable with a simple $\mathrm{MRC}$ receiver, is given by

$$
P_{b, S U}=E\left[Q\left(\sqrt{\frac{2 E_{b}}{N_{0}} \frac{1}{N} \sum_{k \in \Psi_{m}} \sum_{l}\left|H_{k}^{(l)}\right|^{2}}\right)\right]
$$

where the expectation is over all channel realizations and all data symbols. Clearly, $P_{b, M F B}=P_{b, S U}$ when $K=N$; for $K<N, P_{b, S U}$ is typically worse than $P_{b, M F B}$.

Let us first assume that there is no power control at the BS, i.e., all users have the same power (this means that $\xi_{p}$ is constant).

In fig. 3 we compare semi-analytical BER values, given by (43), with simulated ones for a DSCDMA system with $N=K=256$ and $L=1$ (similar behaviors were observed for MC-CDMA systems). Clearly, the semi-analytical BER values are very close to the simulated ones for the first iteration; for the remaining iterations, the theoretical values are slightly optimistic (For $L>1$ the semi-analytical BER values are even closer to the simulated ones.). Fig. 4 shows the evolution of the correlation factor $\rho$, together with the corresponding estimates (given by (42)), using an estimated BER obtained from the SNIR, as in (43). Clearly, the $\rho$ estimates are very close to

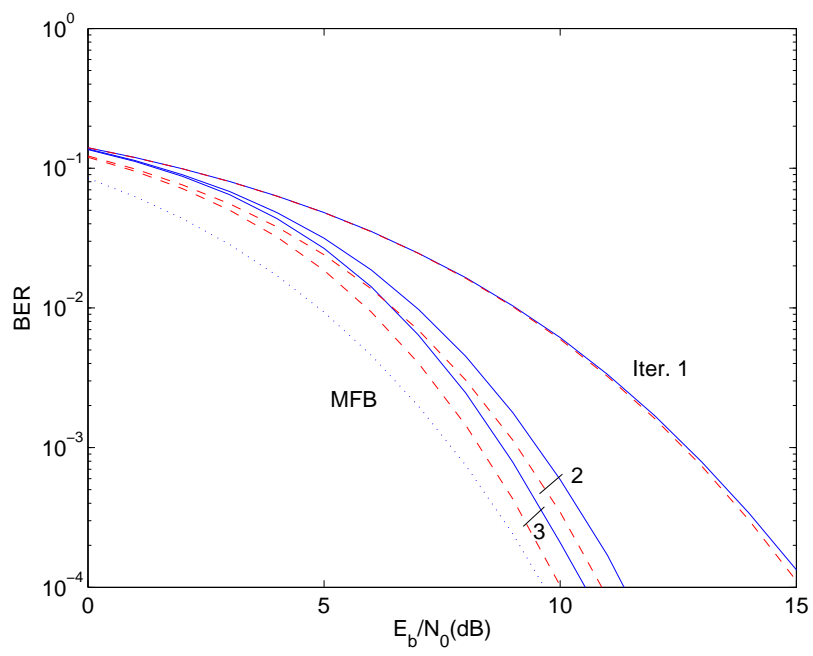

Figure 3. Semi-analytical (dashed line) and simulated (solid line) BER results when $L=1$, for a given number of iterations. 


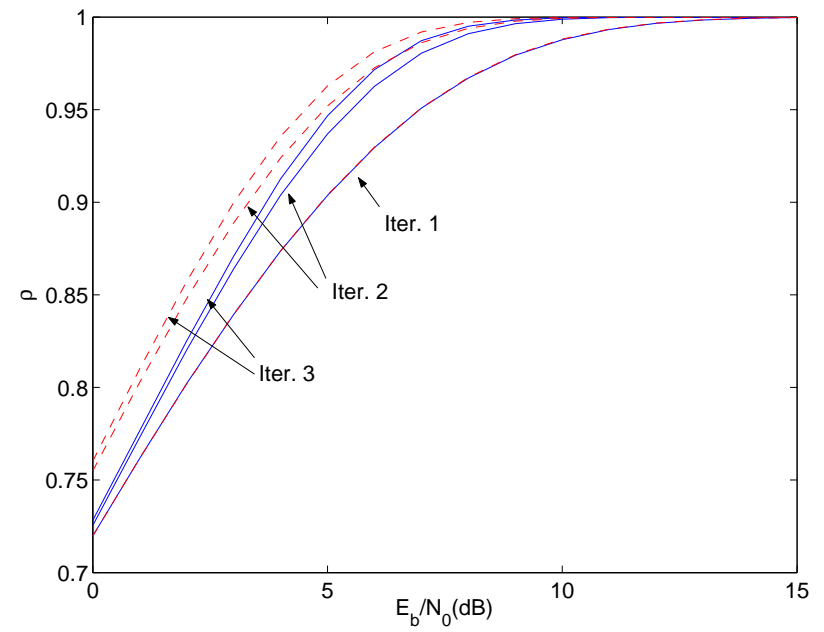

Figure 4. Evolution of $\rho$, when $L=1$ (semi-analytical (dashed line) and simulated (solid line) results).

the true $\rho$ values for the first iteration. When the number of iterations is increased, $\rho$ becomes slightly overestimated when the noise levels are high. For moderate-to-low noise levels, the $\rho$ estimates are still very accurate. The high accuracy of the $\rho$ estimates is a consequence of the approximated BER values given by (43) being close to the true ones (see also fig. 3 ).

Figs. 5 and 6 concern MC-CDMA and DS-CDMA schemes, respectively, once again with $N=K=256$. Clearly, the iterative procedure allows a significant improvement relatively to the conventional linear FDE (first iteration). Moreover, the achievable performance is close to the MFB after three iterations. It was also observed that the performance is similar for MC-CDMA and DS-CDMA schemes. This is not surprising, since for $K=N$ all the available bandwidth is used to transmit each data symbols in both cases.

Let us consider now that $K=P=256$ and the power assigned to $K / 2=128$ users is $6 \mathrm{~dB}$ below the power assigned to the other $K / 2=128$ users. Clearly, the low-power users face strong interference levels. Fig. 7 presents the BER for a DS-CDMA system (similar results were observed for MC-CDMA systems), expressed as a function of the $E_{b} / N_{0}$ of high-power users $\left(6 \mathrm{~dB}\right.$ below the $E_{b} / N_{0}$ of lowpower users). Once again, the iterative receiver allows significant performance improvements. From this figure, it is clear that performance gains associated to the iterative procedure are higher for low-power users

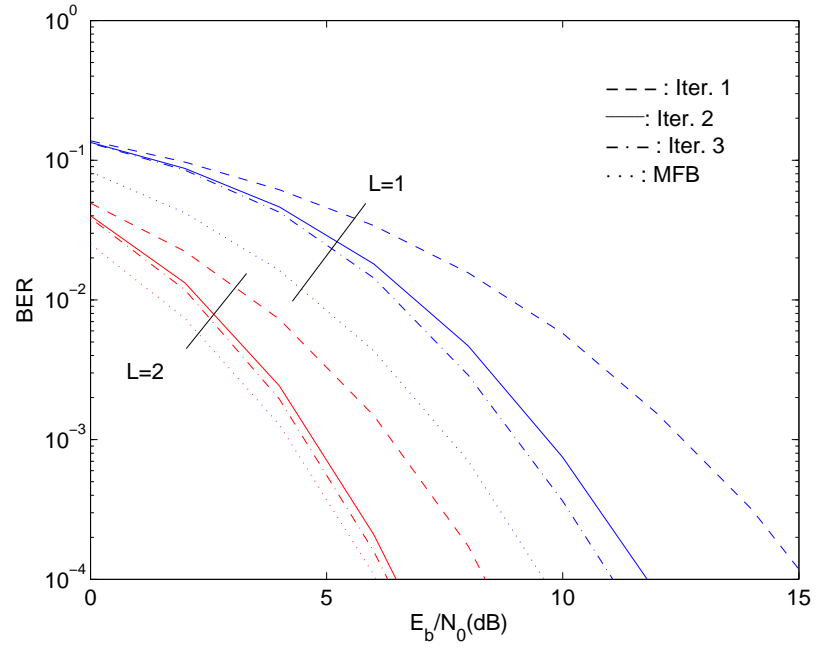

Figure 5. MC-CDMA BER performance when $K=256$ $(M=1)$ and $P=256$ users, with the same assigned power.

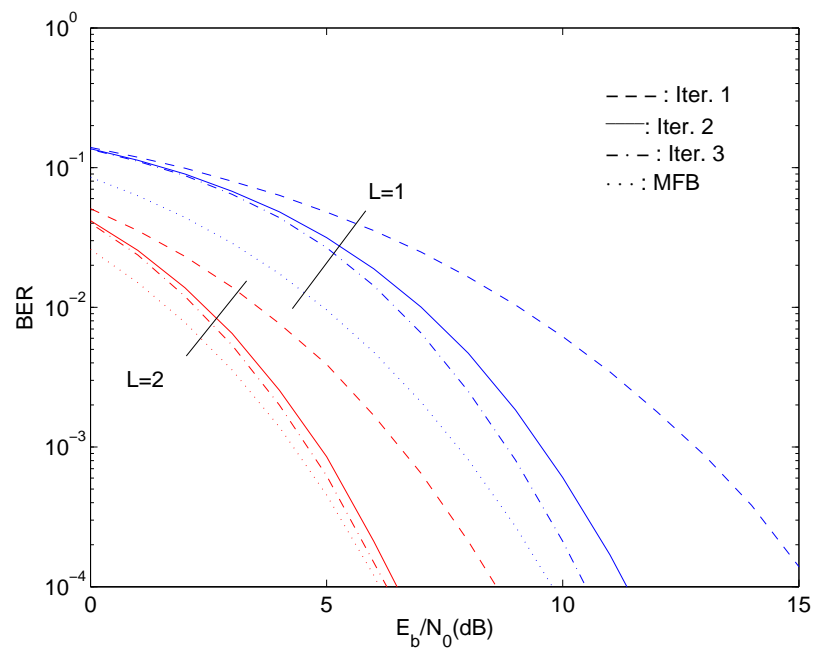

Figure 6. DS-CDMA BER performance when $K=256$ $(M=1)$ and $P=256$ users, with the same assigned power.

and the corresponding BERs are closer to the MFB than for high-power users (the performance of highpower users are still a few $\mathrm{dB}$ from the MFB after three iterations). This is explained as follows: the BER is much lower for high-power users, allowing an almost perfect interference cancelation of their effects on lowpower users; the higher BERs for the low-power users preclude an appropriate interference cancelation when we detect high-power users.

It should also be noted that, for $K<N$, the performance of MC-CDMA schemes is worse, since 


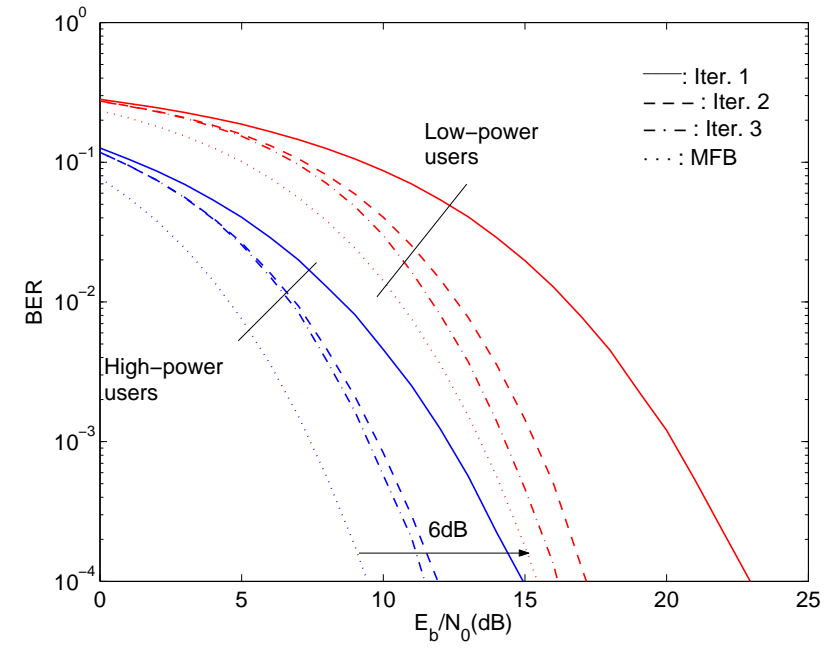

Figure 7. DS-CDMA BER performance with $K / 2=128$ low-power users and $K / 2=128$ high-power users.

just a fraction $1 / M$ of the frequencies is used for the transmission of a given data symbol. This is not the case of DS-CDMA, where all frequencies can be used for transmitting each data symbol, regardless of the spreading factor. For instance, fig. 8 concerns the case where $K=16$ (i.e., $M=64$ ), the same power is assigned to all spreading sequences and we have $P=16$ users (i.e., a fully loaded system). Although the iterative procedure allows gains of about $2 \mathrm{~dB}$, the achievable performance is similar with two or three iteration, and still far from the MFB and the SU performance (the SU performance is slightly worse that the MFB when $K<N)$.

However, it should be noted that this does not mean necessarily an weakness of the MC-CDMA schemes with small spreading factors (small $K$ ). The comparison between DS-CDMA and MC-CDMA schemes should take into account other aspects, such as the envelope fluctuations of the transmitted signals and the impact of the channel coding (one might expect larger coding gains for MC-CDMA schemes, especially when a small $K$ is combined with interblock interleaving).

\section{Conclusions and Complementary Remarks}

In this paper we considered the use of IB-DFE techniques for CP-assisted DS-CDMA and MCCDMA systems. With these IB-DFE techniques, the results of the first iteration correspond to those

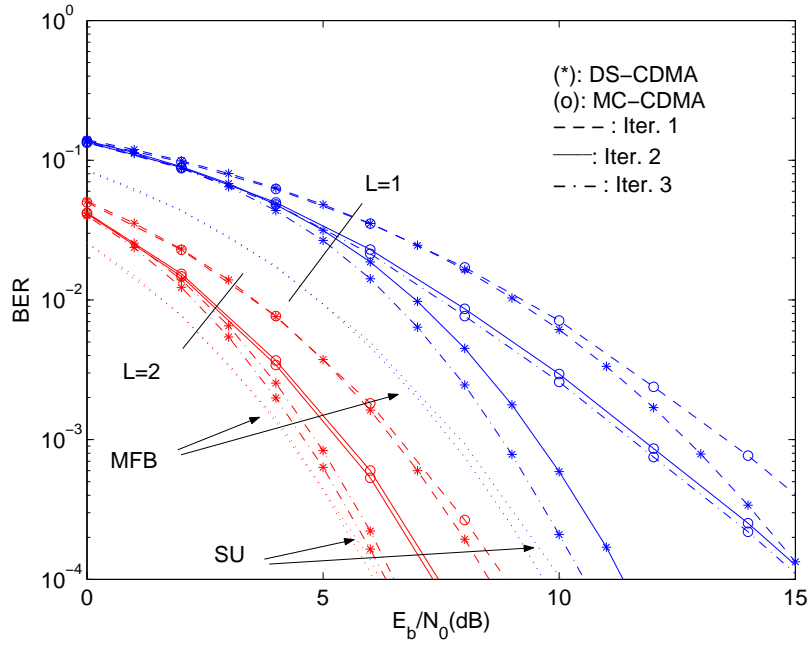

Figure 8. Average BER performance when $K=16(M=$ 64 ) and $P=16$ users, with the same assigned power.

of the conventional, linear, FDE/MMSE technique (Minimum Mean Squared Error); the subsequent iterations provide a performance enhancement, thanks to the iterative cancellation of residual interference. Since the feedback loop takes into account not just the hard-decisions for each block, but also an "overall block reliability", the error propagation problem is significantly reduced. Therefore, the proposed receivers have excellent performance, that can be close to the MFB performance, especially for DS-CDMA schemes. Moreover, their implementation is much less complex than that of receivers employing frequencydomain turbo-equalization [23].

It should be noted that the type of spreading adopted can have a significant impact on the performance of CP-assisted CDMA schemes. As an extreme example (see Fig. 9), for $M=1$, full code usage under equal power conditions and a Fourier spreading/despreading with no complementary scrambling, the MC-CDMA scheme considered in this paper is equivalent to a CP-assisted SC scheme [24] (see fig. 10), and our receiver reduces to the IB-DFE receiver described in [14]. On the other hand, for $M=1$, full code usage under equal power conditions and a Fourier spreading/despreading with no complementary scrambling, the DS-CDMA scheme considered in this paper is equivalent to an OFDM scheme (then there is no advantage in using the IBDFE receiver). 


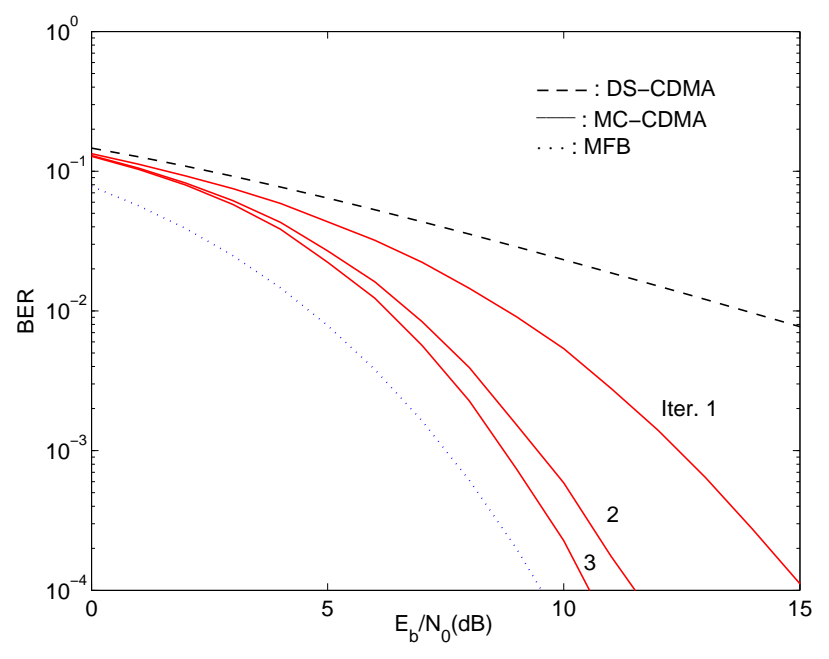

Figure 9. BER performances for a Fourier spreading (with no scrambling), when $N=K=256$ and $P=256$ spreading codes, with the same assigned power.

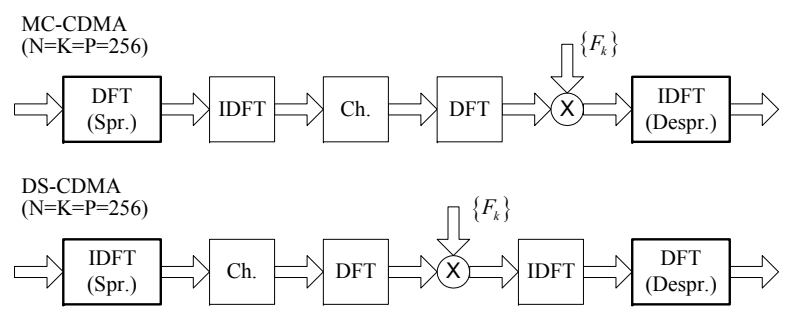

Figure 10. Transmission models for $N=K=P=256$ and Fourier spreading/despreading.

\section{Acknowledgment}

The authors would like to acknowledge the reviewers for their helpful suggestions, which improved the quality of the paper.

\section{REFERENCES}

1. L.Cimini Jr., "Analysis and Simulation of a Digital Mobile Channel using Orthogonal Frequency Division Multiplexing", IEEE Trans. on Comm., Vol. 33, No. 7, July 1985.

2. H.Sari, G.Karam and I.Jeanclaude, "An Analysis of Orthogonal Frequency-division Multiplexing for Mobile Radio Applications", In Proc. IEEE Vehic. Tech. Conf., VTC'94, pp. 1635-1639, Stockholm, June 1994.

3. S. Hara and R. Prasad, "Overview of Multicarrier CDMA", IEEE Comm. Magazine, Dec. 1997.

4. S. Hara and R. Prasad, "Design and Performance of Multicarrier CDMA System in Frequency-Selective
Rayleigh Fading Channels", IEEE Trans. on Vehicular Technology, Vol. 48, No. 5, Sep. 1999.

5. H. Sari, "Orthogonal Multicarrier CDMA and its Detection on Frequency-Selective Channels", European Trans. on Telecomm., Vol. 13, No. 5, pp. 439445, Sep.-Oct. 2002.

6. S. Barbarossa and F. Cerquetti, "Simple Space-Time Coded SS-CDMA Systems Capable of Perfect MUI/ISI Elimination", IEEE Comm. Letters, Vol. 5, No. 12, pp. 471-473, Dec. 2001.

7. K. Baum, T. Thomas, F. Vook, V. Nangia, "CyclicPrefix CDMA: An Improved Transmission Method for Broadband DS-CDMA Cellular Systems", IEEE WCNC, pp. 183-188, 2002.

8. S. Verdu, "Minimum Probability of Error for Asynchronous Gaussian Multiple-Access Channels", IEEE Trans. on Inf. Theory, Vol. 32, No. 1, pp. 85-96, Jan. 1986.

9. S. Verdu, "Computational Complexity of Optimum Multiuser Detection", Algorithmica, Vol. 4, No. 1, pp. 303-312, Dec. 1989.

10. K. Fazel and S. Kaiser, Multi-carrier \& Spread Spectrum Systems, Wiley \& Sons, Sep. 2003

11. A.Gusmão, R.Dinis and N.Esteves, "On Frequencydomain Equalization and Diversity Combining for Broadband Wireless Communications", IEEE Trans. on Comm., Vol. 51, No. 7, pp. 1029-1033, July 2003.

12. N. Benvenuto and S. Tomasin, "Block Iterative DFE for Single Carrier Modulation", IEE Elec. Let., Vol. 39, No. 19, pp. 1144-1145, Sep. 2002.

13. M. Reinhardt and J. Lindner, "Transformation of a Rayleigh Fading Channel into a Set of Parallel AWGN Channels and its Advantage for Coded Transmission", IEE Elect. Letters, Vol. 31, No. 25, pp. 2154-2155, Dec. 1995.

14. R. Dinis, A. Gusmão, and N. Esteves, "On Broadband Block Transmission over Strongly Frequency-Selective Fading Channels", Proc. Wireless 2003, Calgary, Canada, July 2003.

15. R. Kalbasi, R. Dinis, D. Falconer and A. Banihashemi, "Layered Space-Time Receivers for SingleCarrier Transmission with Iterative Frequency-Domain Equalization", IEEE VTC'04 (Spring), Milan, Italy, May 2004.

16. R. Dinis, R. Kalbasi, D. Falconer and A. Banihashemi, "Iterative Layered Space-Time Receivers for SingleCarrier Transmission over Severe Time-Dispersive Channels", IEEE Comm. Letters, Sep. 2004.

17. S. Kaiser and J. Hagenauer, "MC-CDMA with Iterative Decoding and Soft-Interference Cancellation", IEEE GLOBECOM'97, Nov. 1997. 
18. X. Wang and H. Poor, "Iterative (Turbo) Soft Interference Cacellation and Decoding for Coded CDMA", IEEE Trans. on Comm., Vol. 47, No. 7, July 1999.

19. T. Ojamperä and R. Prasad, Wideband CDMA for Third Generation Mobile Communications, Artech House Publ., 1998.

20. A.Gusmão, P.Torres, R.Dinis and N.Esteves, "A Class of Iterative FDE Techniques for Reduced-CP SC-Based Block Transmission", Int. Symposium on Turbo Codes, April 2006.

21. A.Gusmão, R.Dinis, J.Conceição and N.Esteves, "Comparison of Two Modulation Choices for Broadband Wireless Communications", In Proc. IEEE Vehic. Tech. Conf., VTC'2000 (Spring), pp. 1300-1305, Tokyo, Japan, May 2000.

22. ETSI, "Channel models for HIPERLAN/2 in Different Indoor Scenarios", ETSI EP BRAN 3ERI085B, pp. 1-8, March 1998.

23. M. Tüchler, J. Hagenauer, "Linear Time and Frequency Domain Turbo Equalization", IEEE VTC'01 (Fall), Vol. 4, pp. 2773-2777 Oct. 2001.

24. K. Brüninghaus and H. Rohling, "Multi-carrier Spread Spectrum and its Relationship to Single-carrier Transmission", IEEE VTC'98, May 1998. 